\title{
Systematic and quantitative mRNA expression analysis of TRP channel genes at the single trigeminal and dorsal root ganglion level in mouse
}

\author{
Ine Vandewauw, Grzegorz Owsianik and Thomas Voets ${ }^{*}$
}

\begin{abstract}
Background: Somatosensory nerve fibres arising from cell bodies within the trigeminal ganglia (TG) in the head and from a string of dorsal root ganglia (DRG) located lateral to the spinal cord convey endogenous and environmental stimuli to the central nervous system. Although several members of the transient receptor potential (TRP) superfamily of cation channels have been implicated in somatosensation, the expression levels of TRP channel genes in the individual sensory ganglia have never been systematically studied.

Results: Here, we used quantitative real-time PCR to analyse and compare mRNA expression of all TRP channels in TG and individual DRGs from 27 anatomically defined segments of the spinal cord of the mouse. At the mRNA level, 17 of the 28 TRP channel genes, TRPA1, TRPC1, TRPC3, TRPC4, TRPC5, TRPM2, TRPM3, TRPM4, TRPM5, TRPM6, TRPM7, TRPM8, TRPV1, TRPV2, TRPV4, TRPML1 and TRPP2, were detectable in every tested ganglion. Notably, four TRP channels, TRPC4, TRPM4, TRPM8 and TRPV1, showed statistically significant variation in mRNA levels between DRGs from different segments, suggesting ganglion-specific regulation of TRP channel gene expression. These ganglion-to-ganglion differences in TRP channel transcript levels may contribute to the variability in sensory responses in functional studies.

Conclusions: We developed, compared and refined techniques to quantitatively analyse the relative mRNA expression of all TRP channel genes at the single ganglion level. This study also provides for the first time a comparative mRNA distribution profile in TG and DRG along the entire vertebral column for the mammalian TRP channel family.
\end{abstract}

Keywords: Dorsal root ganglia, Trigeminal ganglia, TRP channels, Somatosensation, mRNA expression analysis, Sensory neurons

\section{Background}

Somatosensation describes the ability to detect a wide range of endogenous and environmental stimuli, such as temperature, mechanical forces, chemical stimuli and pain [1]. These stimuli are conveyed by sensory nerve fibres arising from cell bodies within the trigeminal ganglion (TG) in the head and within dorsal root ganglia (DRG), located lateral to the spinal cord in the vertebral column.

\footnotetext{
*Correspondence: Thomas.Voets@med.kuleuven.be

Laboratory of Ion Channel Research, Department of Cellular and Molecular Medicine and TRPLe (TRP Research Platform Leuven), KU Leuven, Leuven, Belgium
}

To understand the mechanisms of somatosensation and nociception, it is important to identify the molecules that contribute to stimulus detection and promote excitation or sensitization of the primary sensory nerve fibres. In this context, a lot of attention has been focused on the cationpermeable ion channels of the transient receptor potential (TRP) family.

Due to their distinct activation mechanisms and biophysical properties, TRP channels are highly suited to function in sensory cells, either as receptors for environmental or endogenous stimuli or as molecular players in signal transduction cascades downstream of metabotropic 
receptors. As such, members of the TRP superfamily are involved in a variety of sensory processes, such as thermosensation, mechanosensation, osmosensation, olfaction, taste, vision and pain perception [2,3].

There are 28 mammalian TRP channels, which are subdivided into six subfamilies based on amino acid sequence homology: TRPA (ankyrin), TRPC (canonical), TRPM (melastatin), TRPML (mucolipin), TRPP (polycystin) and TRPV (vanilloid) [4]. To study their roles in the somatosensory system, it is important to know where and at what level they are expressed. Unfortunately, current expression studies in mammals are heterogeneous with regard to species, applied method, number of included tissues and cell types, and choice of analysed TRP channels. On the other hand, experiments in the past describing the expression profile of TRP channels in DRGs often gave diverging results [5-9]. It should be noted that there is a significant lab-to-lab or even a researcher-to-researcher variability in the segments of the vertebral column from which DRGs are isolated. Moreover, these differences are mostly not reported or taken into account [10-13]. We hypothesized that the reported discrepancy of TRP gene expression studies are, at least partly, due to variations in TRP gene expression depending on the segmental location of the DRG. We therefore set out to analyse the mRNA expression profile of all 28 mammalian TRP channels in TG and DRGs of each individual segment of the vertebral column of the mouse, thereby covering most of the sensory neurons innervating the skin, mucosa and internal organs. The obtained results form a firm basis for further studies on the relevance of TRP channels in sensory neurons in health and disease.

\section{Results}

\section{Optimization of cDNA libraries for quantitative PCR} analysis

RNA quantity and quality was assessed using the Experion Automated Electrophoresis System (BioRad) and revealed almost 2 orders of magnitude higher yield of total RNA extraction from TGs compared to that obtained from single DRGs $(97.1 \pm 20.1 \mathrm{ng} / \mu \mathrm{l}$ and $3.6 \pm 0.3 \mathrm{ng} / \mu \mathrm{l}$, respectively). In both cases the average quality of isolated total RNAs was relatively high reaching the RNA quality indicator (RQI) of about 8 (Additional file 1: Figure S1). Since amounts of total RNA from a single DRG of a defined segment of vertebral column were very low and in many cases expression levels of ion channel genes were rather poor, we fine-tuned the protocol for generation of cDNA libraries from all our samples. The SuperScript VILO cDNA Synthesis Kit (Life Technologies) was the most efficient in cDNA synthesis reaching from 1.5 to 4 units lower $\mathrm{Ct}$ values (Additional file 1: Figure S2 A). Moreover, all cDNA samples from DRGs were subjected to a preamplification step with the
TaqMan PreAmp Master Mix Kit (Life Technologies) that further increased PCR detections as demonstrated by a reduction of $\mathrm{Ct}$ values by $\sim 6$ (Additional file 1: Figure S2 B).

\section{mRNA profiling of TRP genes in mouse TG and DRGs} Complementary DNA samples from TG and DRGs, including (from head to tail) 7 cervical segments (C1-C7), 13 thoracic segments (T1-T13), 6 lumbar segments (L1-L6) and first sacral segments (S1) were assayed using the TaqMan assays for all 28 mouse TRP channel genes and phosphoglycerate kinase 1 (PGK1) and hypoxanthine guanine phosphoribosyl transferase 1 (HPRT1) genes as endogenous controls. For each ganglion, qPCR analysis was performed on 3-4 independent biological samples (i.e. independently isolated ganglia from different mice). Pair-wise comparisons of the mRNA expression data of independently isolated and processed ganglia from the same anatomical location displayed a strong correlation, indicating that TRP channel gene expression patterns were relatively constant between samples from different mice (Figure 1).

Figure 2 shows relative expression levels of the different TRP channel genes in individual ganglia from different mice pooled per every anatomic segment (see also Additional file 1: Figure S3 and Additional file 2 Table S1). A set of 11 TRP channel genes, TRPC2, TRPC6, TRPC7, TRPM1, TRPML2, TRPML3, TRPP3, TRPP5, TRPV3, TRPV5 and TRPV6, invariably showed very low (more than 200-fold lower than the level of PGK1) to undetectable levels of mRNA expression in TG and all tested DRGs. For the other 17 TRP channel genes, mRNA was consistently detected in all tested ganglia, albeit at varying expression levels. In particular, the highest expression levels (between 0.1. and 2-fold relative to PGK1) were found for TRPA1, TRPC3, TRPM7, TRPML1, TRPP2 and TRPV2 (Figure 2 and Additional file 2: Table S1). Using one-way ANOVA analysis, we compared relative mRNA expression of all 17 expressed TRP channel genes in DRGs from different segments (Figure 3). Four TRP channels, TRPC4, TRPM4, TRPV1 and TRPM8, displayed significant variations in mRNA expression between ganglia, suggesting that neurons in ganglia from different anatomical segments, innervating distinct parts of the body, might exhibit different sensory profiles and/or TRP channel patterns.

\section{Discussion}

The TRP superfamily is a functionally diverse group of cation channels with widely diverging functional properties. First, there is a striking diversity in the pore permeability of TRP channels, ranging from non-selective cation permeability to high selectivity for divalent cations [14]. Moreover, there is a daunting variety of stimuli that can regulate the gating of the TRP channels, such as physical stimuli (temperature, voltage, 

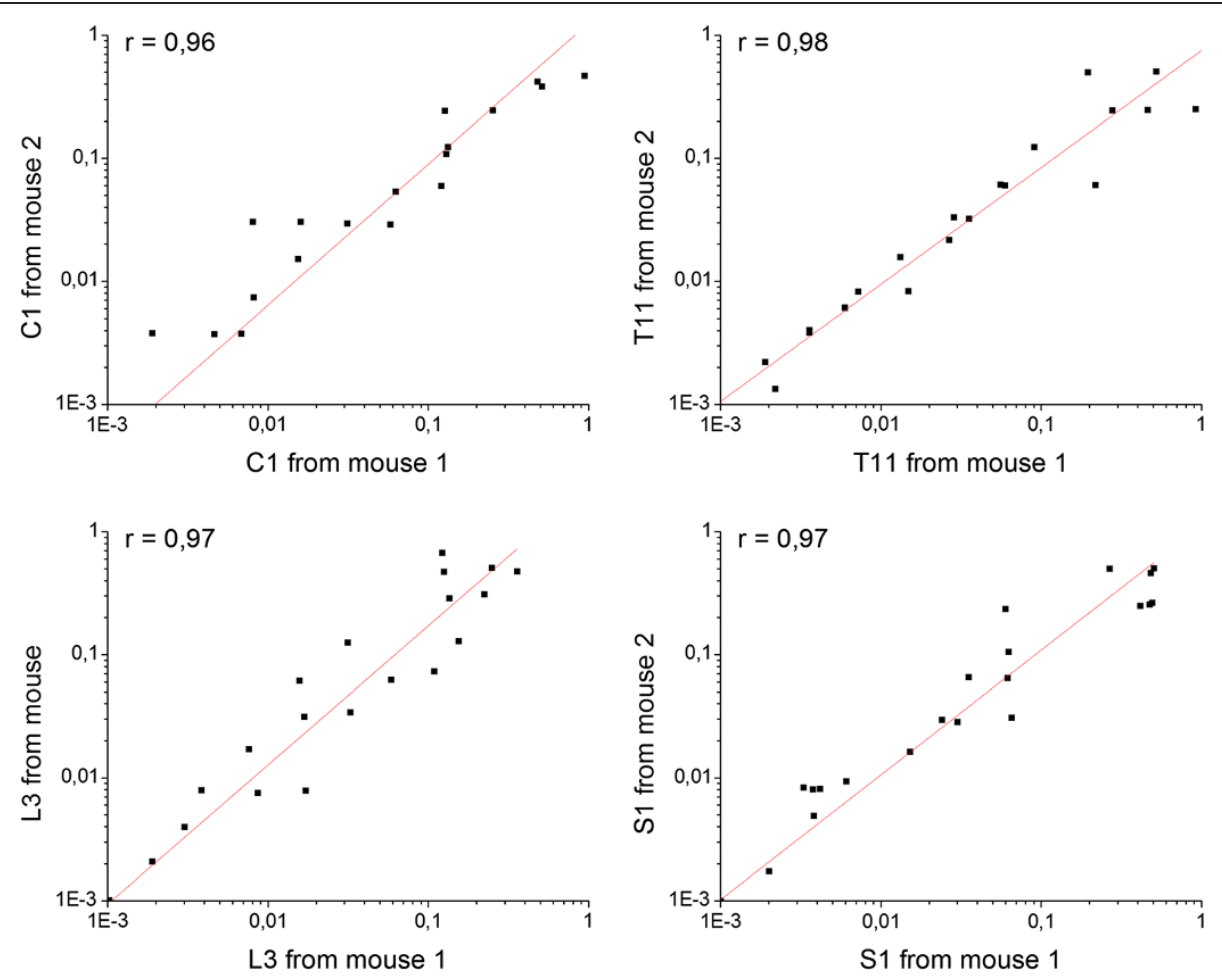

Figure 1 Correlations between relative mRNA expression in different anatomic segments of two mice. Examples of comparison between relative mRNA expressions of all tested mouse TRP channel genes in DRGs from segment C1, T11, L3 and S1 between two mice, using the Pearson correlation analysis.

mechanical stress), exogenous ligands, intracellular cations and lipid components of the plasma membrane [15]. Several TRP channels, including TRPV1, TRPV4, TRPM3, TRPM8 and TRPA1, are implicated in somatosensation, acting as integral molecular components of the sensory machinery involved in chemo, thermo and/ or mechanosensation [16]. Based on experimental data, there was ample evidence for the expression of other TRP channels in sensory neurons, which may either operate as sensors of as yet undefined stimuli or exert other (housekeeping) functions in these cells. The present study had two main aims: (1) to provide a systematic analysis of all TRP channels in different sensory ganglia in mice; and (2) to detect possible ganglion-to-ganglion variations in TRP channel expression. Although destinations of every single DRG neuron projections in mice are still unclear, a homology to human DRG neurons might be instrumental to anticipate TRP channel functions in target organs [17]. In humans, the cervical DRGs mainly projects to dermatomes of the head, upper limbs, hands as well as the eye. The thoracic DRGs innervate dermatomes of the chest and the abdomen (T1-T5 supply the stomach, liver, gallbladder and pancreas while T10-T12 account for the kidneys). The lumbar DRGs innervate dermatomes of the inguinal regions, the anterior and inner surfaces of the lower limbs and feet, while the sacral DRGs project to the remaining posterior and outer surfaces of the lower limbs and lateral margin of the feet (L1-L3 innervate the intestines, L4-S2 are responsible for the innervations of the bladder and prostate, while S3-S5 innervate external genitalia).

We were able to confirm expression of TRP channels that have been previously proposed as sensors in TG and DGR neurons, including TRPA1, TRPV1, TRPV2, TRPV4, TRPM3 and TRPM8 [9,18-22]. Notably, we found that expression levels of TRPV1 and TRPM8 showed statistically significant variation between anatomically different ganglia. For example, TRPM8 expression was significantly lower in the DRG from thoracic segments compared to TG or other regions of the spinal cord, whereas TRPV1 showed significantly higher expression levels in L6 and S1 segments. Such variations may contribute to the variability in functional TRPM8or TRPV1-mediated responses in neurons isolated from different segments.

Amongst the most highly expressed genes there were also a number of TRP channels for which a function in the sensory system is not yet well established (TRPC3, TRPM7, TRPML1 and TRPP2). TRPC3, together with TRPC6, has recently been proposed as a component of a 

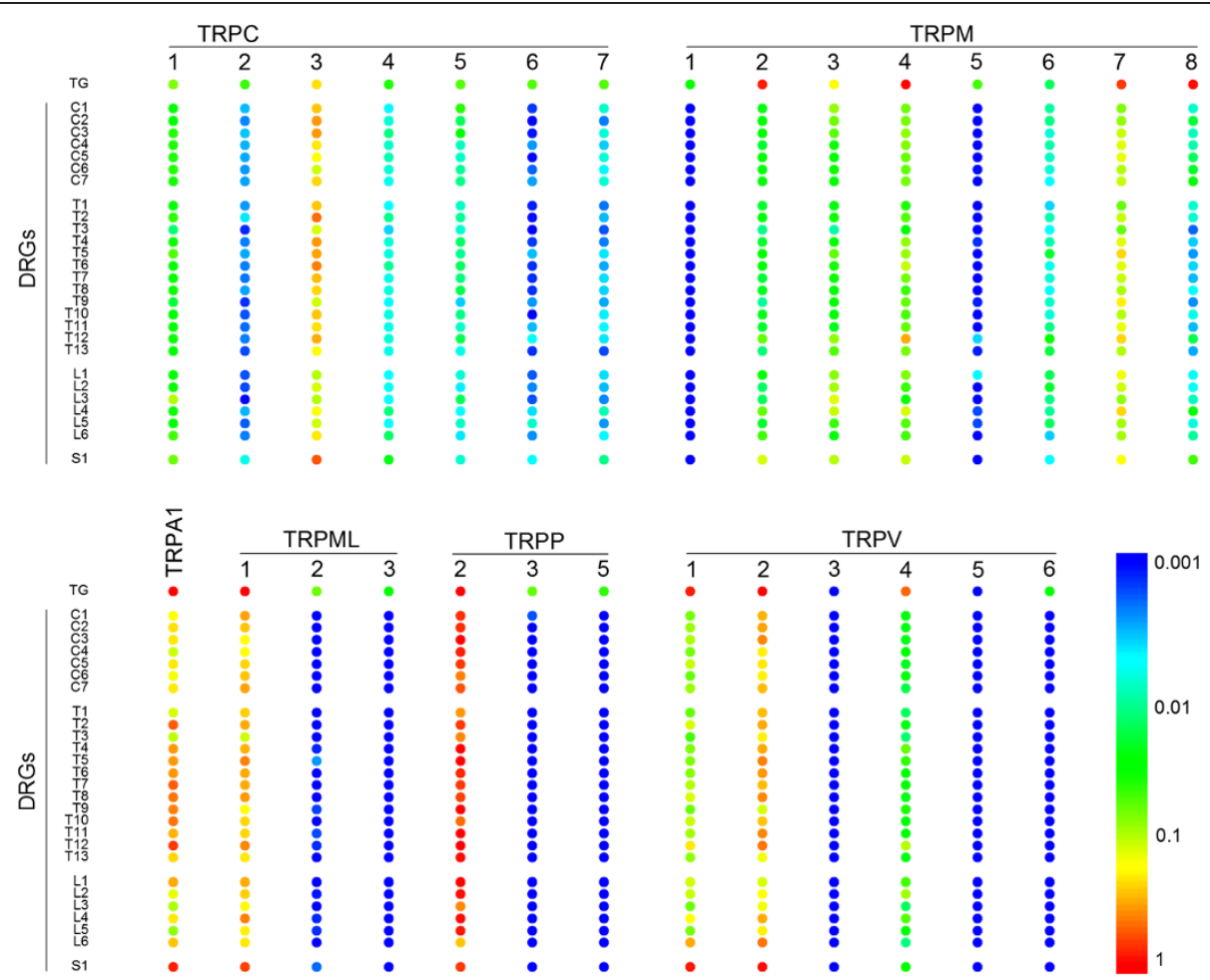

Figure 2 Expression of TRP mRNAs in each individual segment of the vertebral column. Comparison of expression levels of TRP channel genes in samples from isolated TG and DRGs ( $n=3$ or 4). The colour code scale represents the average relative expression from all experiments. All corresponding numerical values are deposed in Additional file 2: Table S1. C1-7 - cervical segments; T1-13 - thoracic segments; L1-6 - lumbar segments; S1 - sacral segment.

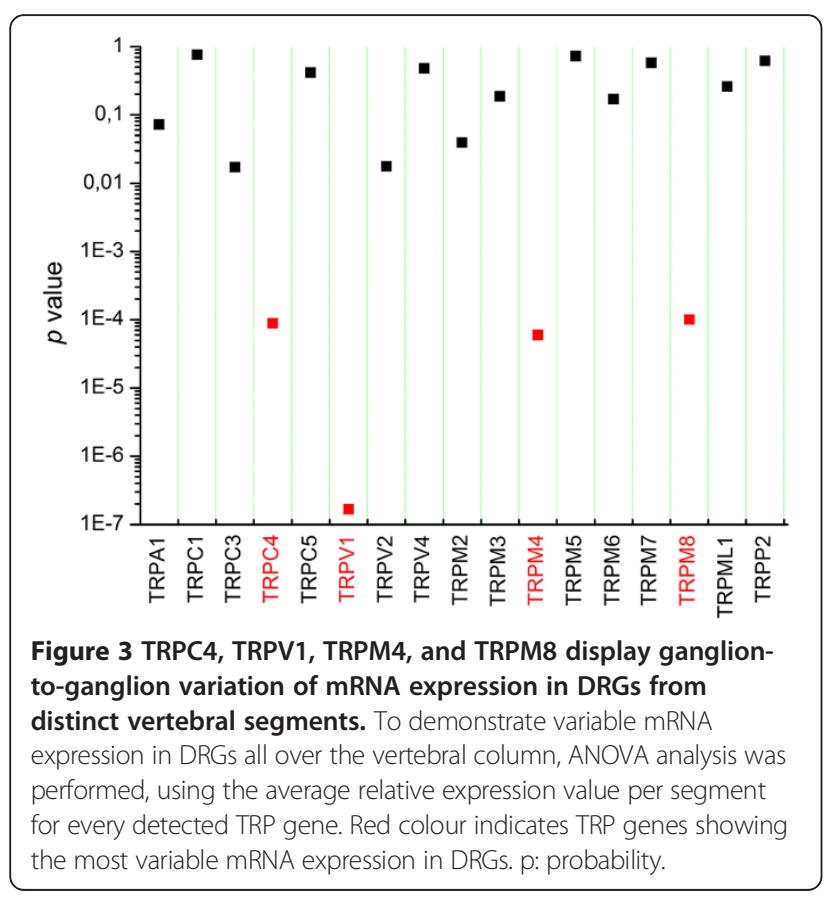

mechanotransduction complex in sensory neurons [23], although there is little evidence that TRPC3 on its own can form a mechano-gated channel. TRPM7 is a ubiquitously expressed channel that has been implicated in cell proliferation, organ development and intracellular $\mathrm{Mg}^{2+}$ homeostasis, although a role in mechanosensation has been suggested in some studies [24,25]. TRPML1 is mainly found in endosomes, and may play a housekeeping role in endosome homeostasis [26]. The function of TRPP2, a TRP channel involved in development of polycystic kidney disease [27], which was found to be the most highly expressed TRP channel gene in the majority of sensory ganglia, is fully unclear.

Expression of 11 TRP channels, TRPC2, TRPC6, TRPC7, TRPM1, TRPML2, TRPML3, TRPP3, TRPP5, TRPV3, TRPV5 and TRPV6, was generally low to undetectable, suggesting that these channels have no specific function in sensory neurons, or only in a very small subset of these cells.

Although these qPCR data provide a valuable comparison of expression at the mRNA level, it is important to remember that mRNA levels not always correlate with the relative abundance of these channels at the protein level or in their functional [28]. Consequently, high 
mRNA levels do not always indicate that these TRP channels are functionally important, and, vice versa, low mRNA expression levels do not exclude the possibility that these TRP channels are relevant in physiology. Thus, systematic studies using TRP subtype-selective antisera could be useful to extend future studies to the protein level that will further help to assess the role of these channels at the functional level.

\section{Conclusions}

In conclusion, we have developed, compared and refined techniques to quantitatively analyse the relative mRNA expression of all TRP channel genes at the single ganglion level using quantitative real-time PCR. This study also provides for the first time a comparative mRNA distribution profile in TG and DRGs along the entire vertebral column for the mammalian TRP channel family.

\section{Methods}

\section{Animals}

WT C57BL/6J male mice from 10-14 weeks of age were used for all experiments. All experiments approved by the KU Leuven Ethical Committee Laboratory Animals (ECD). Approval was granted under project number P021/2012. The authors' laboratory has the Belgian Governmental license for small animal experiments (LA1210551).

\section{RNA extraction and CDNA synthesis}

After euthanasia by $\mathrm{CO}_{2}$ inhalation, TG and DRGs from each segment of the spine were individually removed, washed in ice-cold PBS, put into the RNA later Stabilization Reagent (Qiagen), immediately snap frozen in liquid nitrogen and kept at $-80^{\circ} \mathrm{C}$ until final processing. Total RNA from TG was extracted using the RNeasy Mini Kit (Qiagen), following manufacturer's protocol (for all kits in this study only originally supplied components and protocols were used). RNA concentration and quality was assessed using the Experion RNA StdSens Analysis Kit (Bio-Rad), samples with RQI values below 6 were discarded (Additional file 1: Figure S1) [29]. The total RNA from TG (500 ng) subsequently served for cDNA synthesis using Ready-To-Go YouPrime First-Strand Beads (GE Healthcare). The total RNA from DRGs (10 ng) was extracted using the RNeasy Plus Micro Kit (Qiagen). RNA concentration and quality was assessed using the Experion RNA HighSens Analysis Kit (Bio-Rad), samples with RQI values below 6 were discarded (Additional file 1: Figure S1). The RNA subsequently served for cDNA synthesis using the SuperScript VILO cDNA Synthesis Kit (Life Technologies). Generated cDNAs were stored at $-20^{\circ} \mathrm{C}$ (for the MIQE guidelines checklist see Additional file 3).

\section{RNA preamplification}

cDNA from DRGs was subjected to the TaqMan PreAmp Master Mix Kit (Life Technologies). This kit is intended for use with very small quantities of cDNA (1-250 ng) and increases the quantity of specific cDNA targets for gene expression analysis using TaqMan gene expression assays (Table 1). Preamplification uniformity was assessed using the $\Delta \Delta \mathrm{Ct}$ method, which involves a qPCR with both the original cDNA and the preamplified cDNA as a starting template. A $\Delta \Delta \mathrm{Ct}$ value close to zero indicates that there is preamplification uniformity, with limits set at \pm 1.5 . All target genes produced $\Delta \Delta \mathrm{Ct}$ values within these limits (Additional file 1: Figure S2 B,C), indicating that there is no significant amplification bias.

Table 1 List of TaqMan gene expression assays (life technologies) used in the study

\begin{tabular}{|c|c|c|c|c|c|}
\hline $\begin{array}{l}\text { Gene } \\
\text { Name }\end{array}$ & Assay ID & $\begin{array}{l}\text { GenBank } \\
\text { mRNA }\end{array}$ & $\begin{array}{l}\text { Exon } \\
\text { boundary }\end{array}$ & $\begin{array}{l}\text { Assay } \\
\text { location }\end{array}$ & $\begin{array}{l}\text { Amplicon } \\
\text { length }\end{array}$ \\
\hline TRPA1 & Mm00625268 & AY231177.1 & $7-8$ & 942 & 73 \\
\hline TRPC1 & Mm00441975 & AF191551.1 & $8-9$ & 1546 & 129 \\
\hline TRPC2 & Mm00441984 & AF111107.1 & $15-16$ & 2675 & 77 \\
\hline TRPC3 & Mm00444690 & AF190645.1 & $3-4$ & 1086 & 129 \\
\hline TRPC4 & Mm00444284 & AF011543.1 & $6-7$ & 1934 & 122 \\
\hline TRPC5 & Mm00437183 & AF029983.1 & $5-6$ & 1755 & 105 \\
\hline TRPC6 & Mm01176083 & U49069.1 & $1-2$ & 463 & 65 \\
\hline TRPC7 & Mm00442606 & AF139923.1 & $6-7$ & 1678 & 93 \\
\hline TRPM1 & Mm00450619 & AF047714.1 & $6-7$ & 822 & 90 \\
\hline TRPM2 & Mm00663098 & AB166747.1 & $29-30$ & 4265 & 107 \\
\hline TRPM3 & Mm00616485 & AK051867.1 & $21-22$ & 3135 & 75 \\
\hline TRPM4 & Mm00613173 & AJ575814.1 & $9-10$ & 1204 & 78 \\
\hline TRPM5 & Mm00498453 & AB039952.1 & $18-19$ & 2787 & 73 \\
\hline TRPM6 & Mm00463112 & AK080899.1 & $13-14$ & 1520 & 125 \\
\hline TRPM7 & Mm00457998 & AY032951.1 & $13-14$ & 1752 & 125 \\
\hline TRPM8 & Mm00454566 & AF481480.2 & $24-25$ & 3389 & 89 \\
\hline TRPML1 & Mm00522550 & BC005651.1 & $2-3$ & 316 & 64 \\
\hline TRPML2 & Mm00509841 & AF503575.1 & $1-2$ & 163 & 68 \\
\hline TRPML3 & Mm00460328 & AF475086.1 & $8-9$ & 1095 & 82 \\
\hline TRPP2 & Mm00435829 & AF014010.1 & $1-2$ & 673 & 84 \\
\hline TRPP3 & Mm00619572 & AF271381.1 & $4-5$ & 870 & 74 \\
\hline TRPP5 & Mm00450423 & AF182033.1 & $6-7$ & 1022 & 96 \\
\hline TRPV1 & Mm01246302 & AJ620495.1 & $9-10$ & 1593 & 68 \\
\hline TRPV2 & Mm00449223 & AB021665.1 & $3-4$ & 643 & 95 \\
\hline TRPV3 & Mm00454996 & AF510316.1 & $1-2$ & 42 & 57 \\
\hline TRPV4 & Mm00499025 & AJ296078.1 & $2-3$ & 596 & 56 \\
\hline TRPV5 & Mm01166037 & AK085479.1 & $8-9$ & 1214 & 93 \\
\hline TRPV6 & Mm00499069 & AB037373.1 & $1-2$ & 325 & 63 \\
\hline PGK1 & Mm00435617 & M15668.1 & $5-6$ & 546 & 137 \\
\hline HPRT1 & Mm00446968 & J00423.1 & $6-7$ & 570 & 65 \\
\hline
\end{tabular}




\section{Quantitative real-time PCR}

qPCR reactions $(20 \mu \mathrm{l})$, composed of $2 \mu \mathrm{l}$ cDNA template, Universal TaqMan MasterMix (2x concentrated, Life Technologies), TaqMan assay (20x concentrated, Life Technologies) and $\mathrm{H}_{2} \mathrm{O}$, were performed with the 7500 Fast Real-Time PCR System (Life Technologies). Reactions, ran in triplicate, were incubated at $50^{\circ} \mathrm{C}$ for $2 \mathrm{~min}$ and $95^{\circ} \mathrm{C}$ for $10 \mathrm{~min}$, followed by 40 cycles of $95^{\circ} \mathrm{C}$ for $15 \mathrm{sec}$ and $60^{\circ} \mathrm{C}$ for $1 \mathrm{~min}$. Non-template controls (NTCs) were used as negative controls in every experiment. PGK1 and HPRT1, selected using the geNorm application [30], were used as endogenous controls (see also Additional file 4: Table S2). Data represent relative expression of detected mRNA normalized to PGK1 mRNA, which was used as a calibrator for comparative analysis [31].

\section{Statistical analysis}

Origin software (version 8.6; OriginLab) was used for statistical analysis and data display. Data are represented as mean \pm SEM. One-way ANOVA was used for statistical comparison between groups.

\section{Additional files}

Additional file 1: Figure S1. Examples of quality control

measurements of total RNA extractions from different DRGs. Figure S2. Optimization of cDNA libraries for quantitative PCR analysis. Figure S3. Expression of TRP mRNAs in each individual segment of the vertebral column.

Additional file 2: Table S1. Relative expression levels of the different TRP channel genes in individual ganglia. Expression levels of different mice were pooled per segment and expressed as mean \pm SEM. C1-7 - DRGs in cervical segments; T1-13 - DRGs in thoracic segments; L1-6 - DRGs in lumbar segments; S1 - DRG in sacral segment.

\section{Additional file 3: MIQE guidelines checklist.}

Additional file 4: Table S2. Ct values for the reference mRNA genes of individual DRGs per segment. The mean was calculated of experiments done in triplicate.

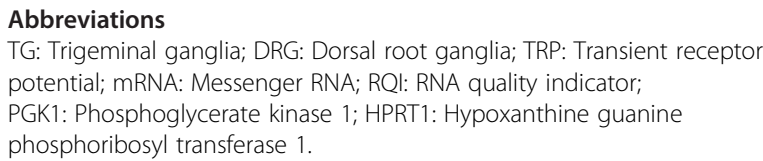

Abbreviations

TG: Trigeminal ganglia; DRG: Dorsal root ganglia; TRP: Transient receptor potential; mRNA: Messenger RNA; RQI: RNA quality indicator; PGK1: Phosphoglycerate kinase 1; HPRT1: Hypoxanthine guanine phosphoribosyl transferase 1.

\section{Competing interests}

The authors declare that they have no competing interests.

\section{Authors' contributions}

IV, GO and TV designed research; IV performed research and analyzed data; IV, GO and TV wrote the paper. All authors read and approved the final manuscript.

\section{Acknowledgements}

We thank all members of the Laboratory of Ion Channel Research for helpful discussions. This work was supported by grants from the Belgian Federal Government (IUAP P7/13), the Research Foundation-Flanders (G.0565.07 and G.0825.11), and the Research Council of the KU Leuven (GOA 2009/07 and PF-TRPLe).
Received: 26 September 2012 Accepted: 31 January 2013

Published: 14 February 2013

\section{References}

1. Lumpkin EA, Caterina MJ: Mechanisms of sensory transduction in the skin. Nature 2007, 445:858-865.

2. Damann N, Voets T, Nilius B: TRPs in our senses. Curr Biol 2008, 18:R880-R889.

3. Voets T, Talavera K, Owsianik G, Nilius B: Sensing with TRP channels. Nat Chem Biol 2005, 1:85-92.

4. Montell C: The TRP superfamily of cation channels. Sci STKE 2005, 2005:re3.

5. Jang Y, Lee $Y$, Kim SM, Yang YD, Jung J, Oh U: Quantitative analysis of TRP channel genes in mouse organs. Arch Pharm Res 2012, 35:1823-1830.

6. Staaf S, Franck MC, Marmigere F, Mattsson JP, Ernfors P: Dynamic expression of the TRPM subgroup of ion channels in developing mouse sensory neurons. Gene Expr Patterns 2010, 10:65-74.

7. Elg S, Marmigere F, Mattsson JP, Ernfors P: Cellular subtype distribution and developmental regulation of TRPC channel members in the mouse dorsal root ganglion. J Comp Neurol 2007, 503:35-46.

8. Hjerling-Leffler J, Alqatari M, Ernfors P, Koltzenburg M: Emergence of functional sensory subtypes as defined by transient receptor potential channel expression. J Neurosci 2007, 27:2435-2443.

9. Vriens J, Owsianik G, Hofmann T, Philipp SE, Stab J, Chen X, Benoit M, Xue F, Janssens A, Kerselaers $S$, et al: TRPM3 is a nociceptor channel involved in the detection of noxious heat. Neuron 2011, 70:482-494.

10. Xu H, Blair NT, Clapham DE: Camphor activates and strongly desensitizes the transient receptor potential vanilloid subtype 1 channel in a vanilloid-independent mechanism. J Neurosci 2005, 25:8924-8937.

11. Dhaka A, Murray AN, Mathur J, Earley TJ, Petrus MJ, Patapoutian A: TRPM8 is required for cold sensation in mice. Neuron 2007, 54:371-378.

12. Story GM, Peier AM, Reeve AJ, Eid SR, Mosbacher J, Hricik TR, Earley TJ, Hergarden AC, Andersson DA, Hwang SW, et al: ANKTM1, a TRP-like channel expressed in nociceptive neurons, is activated by cold temperatures. Cell 2003, 112:819-829.

13. Bodding M, Wissenbach U, Flockerzi V: Characterisation of TRPM8 as a pharmacophore receptor. Cell Calcium 2007, 42:618-628.

14. Owsianik G, Talavera K, Voets T, Nilius B: Permeation and selectivity of TRP channels. Annu Rev Physiol 2006, 68:685-717.

15. Venkatachalam K, Montell C: TRP channels. Annu Rev Biochem 2007, 76:387-417

16. Bandell M, Macpherson LJ, Patapoutian A: From chills to chilis: mechanisms for thermosensation and chemesthesis via thermoTRPs. Curr Opin Neurobiol 2007, 17:490-497.

17. Lee MW, McPhee RW, Stringer MD: An evidence-based approach to human dermatomes. Clin Anat 2008, 21:363-373.

18. Karashima $Y$, Talavera $K$, Everaerts $W$, Janssens A, Kwan $K Y$, Vennekens $R$, Nilius B, Voets T: TRPA1 acts as a cold sensor in vitro and in vivo. Proc Natl Acad Sci USA 2009, 106:1273-1278.

19. Caterina MJ, Schumacher MA, Tominaga M, Rosen TA, Levine JD, Julius D: The capsaicin receptor: a heat-activated ion channel in the pain pathway. Nature 1997, 389:816-824.

20. Caterina MJ, Rosen TA, Tominaga M, Brake AJ, Julius D: A capsaicinreceptor homologue with a high threshold for noxious heat. Nature 1999, 398:436-441.

21. Lee H, lida T, Mizuno A, Suzuki M, Caterina MJ: Altered thermal selection behavior in mice lacking transient receptor potential vanilloid 4. J Neurosci 2005, 25:1304-1310.

22. Bautista DM, Siemens J, Glazer JM, Tsuruda PR, Basbaum Al, Stucky CL, Jordt SE, Julius D: The menthol receptor TRPM8 is the principal detector of environmental cold. Nature 2007, 448:204-208.

23. Quick K, Zhao J, Eijkelkamp N, Linley JE, Rugiero F, Cox JJ, Raouf R, Gringhuis M, Sexton JE, Abramowitz J, et al: TRPC3 and TRPC6 are essential for normal mechanotransduction in subsets of sensory neurons and cochlear hair cells. Open Biol 2012, 2:120068.

24. Fleig A, Penner R: The TRPM ion channel subfamily: molecular, biophysical and functional features. Trends Pharmacol Sci 2004, 25:633-639.

25. Monteilh-Zoller MK, Hermosura MC, Nadler MJ, Scharenberg AM, Penner R, Fleig A: TRPM7 provides an ion channel mechanism for cellular entry of trace metal ions. J Gen Physiol 2003, 121:49-60.

26. Manzoni M, Monti E, Bresciani R, Bozzato A, Barlati S, Bassi MT, Borsani G Overexpression of wild-type and mutant mucolipin proteins in 
mammalian cells: effects on the late endocytic compartment organization. FEBS Lett 2004, 567:219-224.

27. Kottgen M: TRPP2 and autosomal dominant polycystic kidney disease. Biochim Biophys Acta 2007, 1772:836-850.

28. Maier T, Guell M, Serrano L: Correlation of mRNA and protein in complex biological samples. FEBS Lett 2009, 583:3966-3973.

29. Bustin SA, Benes V, Garson JA, Hellemans J, Huggett J, Kubista M, Mueller R, Nolan T, Pfaffl MW, Shipley GL, et al: The MIQE guidelines: minimum information for publication of quantitative real-time PCR experiments. Clin Chem 2009, 55:611-622.

30. Vandesompele J, De Preter K, Pattyn F, Poppe B, Van Roy N, De Paepe A, Speleman F: Accurate normalization of real-time quantitative RT-PCR data by geometric averaging of multiple internal control genes. Genome Biol 2002, 3:RESEARCH0034.

31. Livak KJ, Schmittgen TD: Analysis of relative gene expression data using real-time quantitative PCR and the 2(-Delta Delta C(T)) Method. Methods 2001, 25:402-408.

doi:10.1186/1471-2202-14-21

Cite this article as: Vandewauw et al:: Systematic and quantitative mRNA expression analysis of TRP channel genes at the single trigeminal and dorsal root ganglion level in mouse. BMC Neuroscience 2013 14:21.

\section{Submit your next manuscript to BioMed Central and take full advantage of:}

- Convenient online submission

- Thorough peer review

- No space constraints or color figure charges

- Immediate publication on acceptance

- Inclusion in PubMed, CAS, Scopus and Google Scholar

- Research which is freely available for redistribution 